\title{
The Impact of Sonic Logos on Brand Perceptions: An Abstract
}

\author{
Shawn P. Scott, Daniel Sheinin, and Lauren I. Labrecque
}

\begin{abstract}
Sonic logos have been around since the original NBC chimes, which was the first sound to receive an audio trademark. Even now, Sonic Logos are utilized as a strategic branding element across a variety of mediums. Despite the importance of sonic logos, surprisingly little research has examined their usage and impact on consumer perceptions of brands. Significant work has investigated other forms of music and marketing such as background music (Park and Young 1986), creating the impression of social presence (Sayin et al. 2015), persuasion in marketing (Bruner 1990; Kellaris and Cox 1989; Park 2003), and through the use of sounds and music in retail or public environments (Beverland et al. 2006; Mattila and Wirtz 2001; Morrison and Beverland 2003; Spangenberg et al. 2005).

In our study, we expand on the previous sonic logo research conducted by Krishnan et al. (2012). In their study, they looked at how the number of tones affected consumers' willingness to pay and processing fluency. In our pretest and experiment, we use the baseline results from Krishnan et al. (2012), which showed an optimal six sonic tones and explore how other characteristics of sonic logos, such as timbre, modality, tempo, and key (Bruner 1990), have an effect on consumer perceptions of brands.

In our pretest, we manipulate the characteristics of timbre, modality, tempo, and key that shape consumer evaluations of happiness and sadness in order to create two short (e.g., 4 s) composite orthogonal musical stimuli to use in the remainder of our research. In our experiment, we show that these two separate sonic logos can affect consumers' perceptions of a brand's customer service and attitude toward an advertisement by the mere presence of the sonic logo. In our further experiments, we will continue to tease out various effects that short sonic logos have on consumer sentiment, advertising, and brand perceptions.
\end{abstract}

Keywords Sonic $\log$ os $\cdot$ Sonic branding $\cdot$ Sogos $\cdot$ Branding $\cdot$ Music $\cdot$ Advertising

References Available Upon Request

\author{
S. P. Scott $(\varangle) \cdot$ D. Sheinin · L. I. Labrecque \\ University of Rhode Island, Kingston, RI, USA \\ e-mail: sscott@uri.edu; dsheinin@uri.edu; lauren33@uri.edu
}

(C) The Academy of Marketing Science 2020

S. Wu et al. (eds.), Marketing Opportunities and Challenges in a Changing Global Marketplace, Developments in Marketing Science: Proceedings of the Academy of Marketing Science, https://doi.org/10.1007/978-3-030-39165-2_98 